\title{
Traduire
}

Revue française de la traduction

$220 \mid 2009$

Organisations internationales | Bicentenaire de Louis

Braille

\section{Braille et la musique}

David Ar Rouz

\section{(2) OpenEdition \\ Journals}

Édition électronique

URL : http://journals.openedition.org/traduire/386

DOI : 10.4000/traduire.386

ISSN : 2272-9992

Éditeur

Société française des traducteurs

Édition imprimée

Date de publication : 15 juin 2009

Pagination : 43-56

ISSN : 0395-773X

\section{Référence électronique}

David Ar Rouz, «Braille et la musique », Traduire [En ligne], 220 | 2009, mis en ligne le 12 novembre 2013, consulté le 19 avril 2019. URL : http://journals.openedition.org/traduire/386 ; DOI : 10.4000/ traduire.386 


\section{Braille et la musique}

\section{David ar Rouz}

La musique, universelle ? Voir ! II n'est qu'à écouter une Suite pour violoncelle de Bach sur des enceintes fabriquées par un maître chinois, chanteur d'opéra, pour comprendre qu'à l'autre bout de la planète, ils n'entendent décidément pas comme nous : pour faire court, voire simpliste, ils aiment les sons " acides " quand nous avons tendance à chercher d'abord des basses rondes. Et combien de styles de musique, même sans aller chercher les pays les plus lointains, sommes-nous incapables d'apprécier, parce que notre goût s'est formé au contact de styles trop différents ? Exit donc la belle illusion (bien française ?) de l'universalité...

Alors si on s'intéresse à l'outil de transmission que constitue l'écriture musicale, doit-on s'étonner qu'une traduction puisse être nécessaire ? On peut d'abord penser à l'opération de traduction à laquelle s'entraînera l'élève qui apprend le solfège. Instrumentiste, il aura encore besoin du secours d'un traducteur plus expérimenté, son professeur, qui lui expliquera comment faire passer telle ou telle nuance, tel ou tel détaché, pour donner à son expression un tour plus agréable.

Car, de même que l'acteur doit « interpréter » le texte de théâtre, se l'approprier pour le rendre vivant, l'incarner dans un échange avec le public, le musicien doit aussi faire la partition sienne, avec son bagage musical et son vécu personnel, émotionnel et corporel, pour partager le langage musical avec son public. D'où sans doute la notion si répandue d'" interprète " pour parler des chanteurs et instrumentistes. Et nous ne serons pas les premiers à faire le parallèle entre traduction et interprétation musicale :

Les traductions continuent ou achèvent les textes-source, dont elles sont pour ainsi dire l'exécution - certaines en mauvaise part, il est vrai, comme s'il s'agissait de "l'exécution " d'un texte " condamné ", mais aussi combien d'autres au sens éminent où un grand interprète " exécute " un morceau de musique ! (Ladmiral, 1994, 232)

Mais certains musiciens doivent avoir recours à une autre traduction, d'écrit à écrit, pour faire l'apprentissage de la musique ou simplement élargir leur répertoire : ils sont aveugles. 


\section{D'une écriture à une autre}

Pour Jean Gagnepain, "la moindre conversation est déjà une traduction " (Gagnepain, 1994, 147). En effet, chacun de nous s'approprie la capacité humaine du langage à sa façon et en fonction de son environnement. Mais les différences sont quasiment imperceptibles tant qu'elles ne sont pas handicapantes. On percevra donc plus facilement la différence entre les parlers d'un Français et d'un Allemand qu'entre ceux d'un Québécois et d'un Marseillais. Pourtant, ce n'est qu'une question d'échelle. Et rien n'empêche d'aller plus loin :

Le problème de la traduction, vous le vivez en permanence, dans un ménage par exemple. D'où viennent les scènes de ménages, sinon d'erreurs de traduction ? Personne ne dit exactement ce que dit l'autre. Lorsque les mêmes mots sont dits, aucun des interlocuteurs ne les prend exactement comme les prend l'autre. (ibid., 151)

On peut cependant faire une distinction d'importance entre ces petites traductions que nous opérons tous les jours et l'échange qui nécessite que nous fassions appel à un tiers. II pourra alors s'agir de services de médiation familiale (pour résoudre parfois les erreurs commises dans nos traductions quotidiennes, justement !), d'interprétation, de traduction, de transcription. Dans tous les cas, on aura un ou plusieurs donneurs d'ordre, un prestataire et un ou plusieurs destinataires. Que l'opération se fasse à l'oral ou au moyen de l'outil que constitue l'écriture ne change donc rien à sa nature essentiellement sociale : la traduction sert à surmonter des différences entre des appropriations sociales de la capacité de langage.

En cela, la transcription musicale en Braille est bien une traduction. Car noires, blanches, croches ou rondes, écrites " en noir ", resteront muettes au musicien qui ne voit. II a donc bien besoin de l'intervention d'un tiers, voyant, pour lire la musique, en particulier la musique dite savante. C'est ce à quoi travaille à plein temps Marie-Andrée Courjault au sein de deux associations : l'Association Valentin Haüy (AVH) à Paris et le Centre de transcription musicale Braille par informatique (CTMBI) à Saint-Nazaire. Pianiste, titulaire d'une licence de musicologie, elle maîtrisait donc très bien, avant de se lancer dans l'aventure de la transcription, le langage musical et son écriture.

L'écriture... : formidable outil de communication et de transmission! Car si Mozart avait « tout dans la tête ", il lui était impossible d'interpréter à lui tout seul ses symphonies, il lui fallait bien s'associer des musiciens et leur indiquer ce qu'il attendait d'eux. Et encore la partition n'achevait-elle pas le travail de transmission : il fallait répéter, compléter les indications écrites, expliquer l'idée, ajuster, corriger... Ce qu'on fait encore aujourd'hui : les partitions publiées sont "révisées ", annotées par de grands instrumentistes ; des chercheurs aident les musiciens à s'approcher des interprétations auxquelles les partitions conduisaient au moment de leur composition et, dans le même esprit, des luthiers élaborent des instruments dits " d'époque " pour les armer d'outils qui modifieront l'interprétation des œuvres anciennes ; les compositeurs contemporains, eux, donnent parfois en début de partition une forme de glos- 
saire de leur écriture et des nouveaux symboles qu'ils utilisent, écrivent des indications en toutes lettres au-dessus de la portée... Et toujours le même travail de "répétition "...

Comment pourrait-on s'en passer ? L'écrit a ses limites. La parole, comme la musique, peut être spontanée et créative, fuyante, changeante, polymorphe, infinie. L'écrit évolue beaucoup plus lentement, il est inévitablement limité dans le temps et dans l'espace ; fruit de conventions et de compromis, il exprime rarement toute la pensée de l'écrivain. D'ailleurs, on se rend compte que les compositeurs baroques, par exemple, écrivaient selon les conventions musicales de leur époque et que leurs écrits, à l'instar de ceux d'un Rabelais ou d'un Shakespeare, nécessiteraient presque une " traduction intra-linguistique " pour être interprétés comme ils l'étaient à l'époque par les musiciens d'aujourd'hui. La solution adoptée de préférence consiste à se référer à des traités expliquant les formes anciennes de l'écriture musicale, tels que celui de Jean-Claude Veilhan, Les règles de l'interprétation musicale à l'époque baroque.

En fait, le constat serait à rapprocher de la loi du moindre effort qui prévaut également dans le langage oral : les compositeurs écrivaient le minimum. Avec le temps, quand les goûts changent, à mesure que les compositeurs innovent, l'écriture peut se complexifier. Et l'écriture musicale est certainement complexe. Pas question ici d'infliger un cours de solfège à nos lecteurs! En revanche, nous pouvons comparer l'écriture musicale " en noir " et l'écriture musicale en Braille.

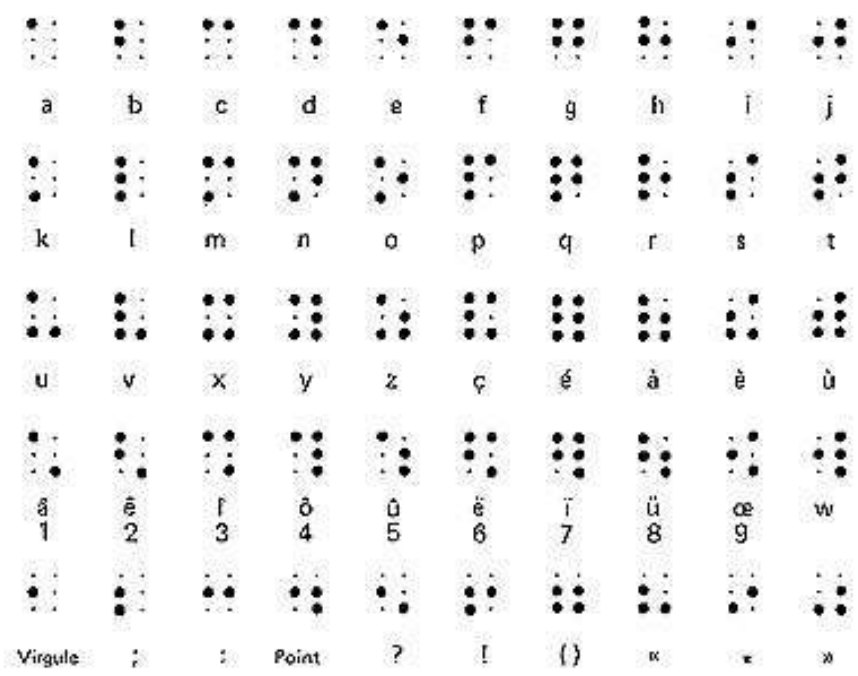

Figure 1: L'alphabet Braille 
Au préalable, il nous faut expliquer un peu ce qu'est le système braille, qui porte le nom de son inventeur, Louis Braille, dont nous fêtons cette année le bicentenaire de la naissance. Aveugle dès l'âge de trois ans suite à une blessure, il entre en 1819 à l'Institut royal des jeunes aveugles, apprend à lire selon la méthode de Valentin Haüy, en lettres gaufrées, c'est-à-dire une impression en relief des caractères d'imprimerie, qui ne permettait qu'une lecture difficile et peu précise, puis s'intéresse à la méthode sonographique d'un certain Charles Barbier de la Serre, qui avait conçu à des fins militaires un système d'écriture au moyen de points en relief. Louis Braille améliorera ce système en réduisant le nombre de points de douze à six, afin que chaque caractère tienne sous le bout des doigts, et le développera pour qu'il exprime, en plus de l'alphabet, la musique dont il fera son métier. II jouait en effet du piano, de l'orgue et du violoncelle.

II utilisera ainsi les caractères suivants pour indiquer les notes et leurs valeurs :

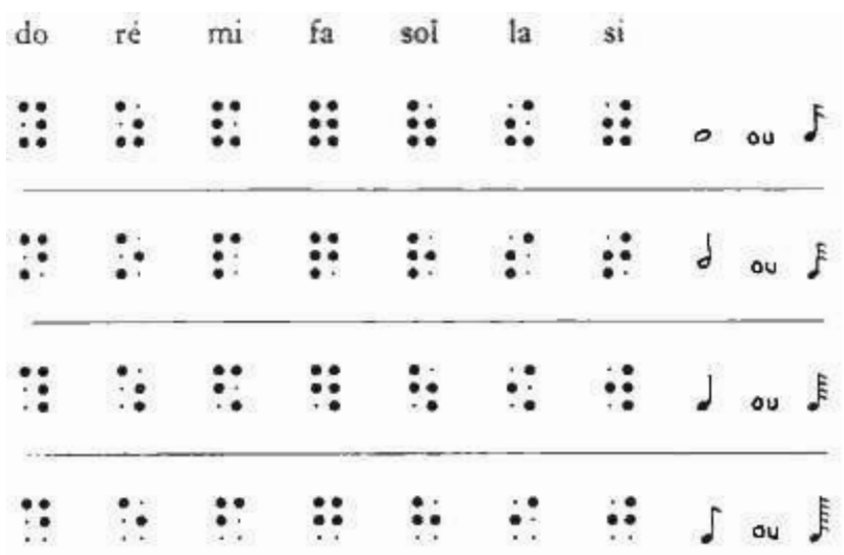

Figure 2 : Notes et valeurs en Braille 
Considérons maintenant l'exemple de réalisation suivant :

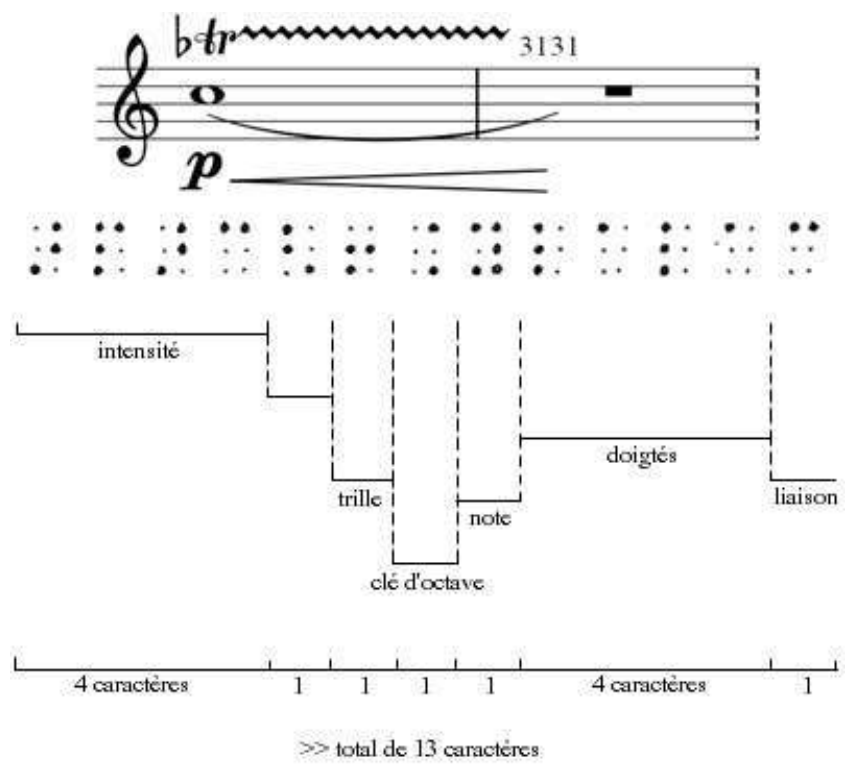

Figure 3 : Exemple de réalisation

On sera tenté tout d'abord de constater qu'il faut treize caractères pour transcrire une seule note en Braille. Ce serait toutefois aller un peu vite en besogne. On peut considérer en effet que la partition en noir compte également treize caractères : un pour la clé de sol qui correspond plus ou moins en Braille à la clé d'octave associée aux notes, deux pour la note elle-même qui comprend une indication de durée et une indication de hauteur matérialisée par la portée, deux pour la nuance (" $\mathrm{P}$ " pour piano et le symbole qui suit pour le crescendo), contre quatre en Braille, le bémol qui précède le trille, lui-même indiqué par deux caractères, le " tr " plus la vaguelette qui suit, quatre pour le doigté, comme en Braille, un pour la liaison.

La différence ne réside donc pas dans la complexité de l'écriture, même si, dans d'autres exemples, on pourra éventuellement constater un certain foisonnement, mais bien plutôt dans la hiérarchisation de l'écriture en noir qui permet de lire le message partiellement. Le musicien voyant pourra ainsi se contenter de lire dans un premier temps la mélodie, en faisant abstraction de toutes les ornementations, des nuances, des liaisons, etc. Le musicien aveugle n'aura pas cette possibilité.

Pour autant, on ne peut pas considérer que l'écriture braille est plus limitée ou limitative que l'écriture en noir. Certes, l'apprentissage du solfège, déjà souvent perçu comme une contrainte 
par bon nombre de débutants, est encore plus contraignant pour le musicien aveugle puisqu'il doit apprendre tous les signes musicaux qu'il est susceptible de rencontrer (en fonction de son instrument) avant de pouvoir lire une partition, aussi simple soit-elle. Mais le mode d'apprentissage qu'il doit adopter, à savoir la mémorisation, segment par segment, de l'intégralité de la partition, le libère ensuite : il peut être tout à la musique, à la technique de son instrument, à son expression...

Quiconque joue d'un instrument sait que le processus d'apprentissage et d'exécution d'un morceau passe par le solfège et la lecture de la partition. Mais il sait aussi que tant que son corps ne pourra pas "penser " la musique et qu'il pensera uniquement consciemment sa relation à l'instrument, ce seront les voisins qui souffriront... (Benasayag et al., 2000, 119).

On ne retrouve pas systématiquement, dans les enseignements de musique dite "classique ", le recours à la mémoire comme moyen de faire progresser les élèves. En revanche, on peut régulièrement constater que ces apprentis musiciens se sentent perdus sans partition et, même après plusieurs années de pratique de leur instrument, n'ont aucune clé pour apprendre l'improvisation, par exemple. Et pour cause : on leur a appris à écrire avant d'apprendre à parler!

Confondre écriture et langage, en effet, c'est confondre les panneaux et le code de la route, le nombre avec le chiffre, la musique avec la notation musicale. Chacun sait bien les méfaits d'un excès de solfège quand l'oreille, d'abord, n'a pas été formée. (Gagnepain, 1990, 230).

Une solution consiste parfois alors à passer par les musiques dites " traditionnelles ", souvent transmises oralement (et non par le solfège et la partition que Benasayag et Sztulwark semblent juger indispensables) pendant très longtemps, souvent répétitives et qui facilitent donc à la fois la mémorisation et, dans un deuxième temps éventuel, l'improvisation. Comme ce ne sont pas les caractéristiques premières de la musique savante, loin de là, l'écriture reste bien sûr nécessaire, et par conséquent sa transcription pour les musiciens aveugles aussi.

\section{La transcription comme traduction}

Outre le fait que la transcription de la musique de noir en Braille peut se définir comme un service de traduction parce qu'il s'agit bien de passer d'un système d'écriture à un autre, s'adressant tous deux à des publics distincts, les similitudes avec l'opération de traduction d'une langue à une autre sont frappantes. Mais avant de les détailler, on note que Marie-Andrée Courjault travaille en fait vers le système d'écriture qui lui est le moins naturel, vers la langue étrangère, si l'on veut. Comment pourrait-il en être autrement ? II faut bien que le transcripteur voie la partition à transcrire! 
Comme tout bon traducteur cependant, surtout s'il travaille vers une langue qui n'est pas sa langue maternelle, mais idéalement aussi quand il traduit dans sa langue maternelle, qu'il en a le temps et les moyens, Marie-Andrée fait relire son texte par son mari, Marcel Courjault, professeur de musique non-voyant. Première analogie.

Après cette spécialité de "langues ", Marie-Andrée peut faire valoir également une spécialité technique. Par rapport à la majorité des transcripteurs de Braille, elle travaille en effet sur un contenu particulier, la musique, où la simple saisie de caractères braille au lieu de caractères d'imprimerie fait place à une musicographie complexe. Elle travaille en outre par informatique, comme l'indique le nom de l'association qui l'emploie, le Centre de transcription musicale Braille par informatique (CTMBI), et utilise un logiciel de saisie du Braille qui permet d'utiliser six touches d'un clavier AZERTY à presser simultanément pour taper les points pertinents du caractère voulu. Les traducteurs, eux, diront qu'ils font du doublage, du sous-titrage, utilisent la TA0, traitent plutôt du rédactionnel, du juridique, traduisent des livres pour enfants, ou toute autre spécialité "technique ".

À propos de technique et de logiciels, on note en outre que les recherches pour la transcription automatique semblent en être au même point que pour la traduction automatique. Si on arrive à peu près à numériser et à transcrire un texte en noir, qui nécessite ensuite une bonne relecture, on parvient à peine pour la musique à transcrire une mélodie simple. II semble peu probable aujourd'hui qu'on puisse se passer dans un avenir proche de l'intervention humaine.

Enfin, Marie-Andrée a aussi ce que j'appellerai une "spécialité de domaine ". Étant pianiste, elle possède nécessairement une meilleure connaissance des partitions écrites pour claviers et des symboles qui y sont utilisés.

Cela ne la dispense pourtant pas de faire des recherches. En effet, la musique contemporaine demandera parfois qu'elle se documente sur le compositeur et son langage propre ou qu'elle rencontre des interprètes qui le connaissent. À plus forte raison si elle transcrit des partitions pour des instruments qu'elle connaît moins, il lui faudra rencontrer par exemple des violonistes pour comprendre les signes particuliers à leur instrument. La guitare, très difficile à transcrire, exigera ainsi des recherches et des relectures attentives. Autrement dit, elle fait appel, à l'instar du traducteur-terminologue quand il veut sortir de sa tour d'ivoire désormais informatisée, à des informateurs, à ceux qui sont au contact quotidiennement avec le langage (écrit, en l'occurrence) qu'elle a à traduire ou avec celui dans lequel elle doit le transcrire...

Et si le système braille est relativement homogène, il n'en reste pas moins que deux transcripteurs pourront aussi faire des choix différents, par exemple concernant les voix qui passent d'une portée à l'autre sur des partitions de piano. Leur formation, plus ou moins axée sur l'analyse musicale, leur fera prendre des voix... pardon, des voies distinctes! Ce sont des détails, bien sûr, tout comme les conventions de présentation adoptées d'un pays à l'autre qui divergent légèrement, mais ils ont leur importance lorsque les lecteurs s'habituent à les lire. 


\section{Questions de marché}

Pas étonnant, donc, que Marie-Andrée soit occupée à plein temps à la transcription de partitions en Braille. Presque un sacerdoce, finalement, car seule une moitié de ce temps de travail lui est rémunérée. Employée 40 heures par mois par l'Association Valentin Haüy, elle travaille le reste du temps pour le CTMBI qui parvient à lui payer 25 heures par mois sous forme de " chèque emploi associatif ", grâce à des subventions qu'il faut solliciter régulièrement. Et malgré cette prise en compte réduite de la charge de travail, l'association reste déficitaire.

Elle parvient toutefois à couvrir ses frais de fonctionnement (téléphone, déplacements, courriers), ainsi que les fournitures (papier, enveloppes...) par les ventes de partitions. Seulement, il n'est pas possible de faire supporter aux clients le prix de revient de la page transcrite, et encore moins le coût exorbitant du matériel quand une imprimante spéciale appelée embosseuse s'achète par exemple 6000 euros l'unité. Pour couvrir ces investissements et la rémunération de la transcriptrice, il faudrait multiplier le prix de la page en Braille par dix. La transcription musicale prend en effet beaucoup de temps, puisque Marie-Andrée produit dans le meilleur des cas quatre pages à l'heure. Or, la page est actuellement vendue un euro jusqu'à 39 pages, avec un minimum forfaitaire de dix euros, puis décroît en fonction du volume de l'ouvrage commandé.

Ce qui nous ramène à une réalité économique qu'on a parfois tendance à oublier, et qui confère pourtant à chacun d'entre nous un réel pouvoir : la loi de l'offre et de la demande. C'est elle, en effet, qui permet de diminuer les coûts de production à mesure que la demande augmente. Pour ne prendre qu'un seul exemple, c'est parce que nous avons été de plus en plus nombreux à acheter des imprimantes que leur coût a fortement diminué, à tel point qu'aujourd'hui, une des actions commerciales de certains fabricants consiste à en offrir ! (À condition quand même qu'on passe la première commande de cartouches chez eux, ce qui pose la question du commerce juteux des consommables... Et tant qu'on y est, il faudrait aussi parler de la dérive qui consiste à diminuer les coûts au maximum, au détriment des emplois sur un marché donné et des conditions de travail sur un autre marché, pour augmenter les profits de quelques-uns.)

Sur le marché qui nous occupe ici, la demande est faible, très faible même. Le CTMBI tient un fichier de 370 clients potentiels, dont 280 ont passé au moins une commande. Cette clientèle est répartie sur tout le territoire hexagonal, avec même quelques clients à l'étranger. MarieAndrée Courjault estime que ce fichier représente peut-être la moitié des musiciens aveugles ou malvoyants français. Le noyau de clients réguliers est constitué essentiellement de professeurs de musique indépendants ou exerçant en écoles de musique. D'autres sont étudiants en musicologie. Et un public prend une importance croissante, à savoir les enfants en écoles, qui requièrent de la part de la transcriptrice une adaptation, puisqu'elle n'utilise pas alors l'abrégé braille et adapte les partitions. 
On comprend donc aisément qu'une imprimante Braille soit chère quand on sait que la France compte moins de 2 millions de personnes aveugles et malvoyantes (chiffres de l'AVH), environ $3 \%$ de la population totale, que ce n'est pas le genre de matériel dont tout le monde a besoin, ni qu'on achète tous les ans, surtout quand le prix en est si élevé. Ce dernier le réserve donc presque automatiquement à des associations ou à des professionnels. Et le public, les clients potentiels ne sont pas plus à même de supporter intégralement le coût de leur travail que d'acheter le matériel pour eux-mêmes.

Si l'on estime le budget moyen d'une association comme le CTMBI, positionnée en outre sur un créneau qui ne concerne directement qu'une infime portion de la population, à $40000 €$, rémunération (faible) d'un salarié à plein temps, charges et frais compris, il faudrait en effet que chaque personne du fichier lui achète en moyenne pour $110 €$ par an de partitions pour qu'elle atteigne l'équilibre. Peut-être un musicien professionnel dépense-t-il cette somme, voire bien davantage, mais un musicien amateur, même ceux qui lisent en noir, probablement jamais.

Les frais doivent donc être supportés par la collectivité et principalement par le mécénat. Estce aberrant ? Non, car la musique crée du lien social, les musiciens aveugles peuvent y contribuer aussi bien que les autres musiciens, voire mieux en raison des conditions de leur travail musical évoquées à la fin de notre première partie. Y aurait-il un autre fonctionnement possible? Peut-être. Aujourd'hui, les transcripteurs demandent une autorisation aux éditeurs de partitions, qui la plupart du temps ne réclament pas de droits pour leur transcription en Braille. Certains l'encouragent même, d'autres y sont totalement indifférents. Pourtant, un professeur de musique aveugle a rarement des élèves aveugles. Ils sont même sans doute en minorité. Et s'il trouve une méthode intéressante, il est susceptible d'inviter ses élèves à l'acheter, en noir cette fois, pour l'utiliser dans leur pratique quotidienne. Ne pourrait-on évaluer les retombées économiques qu'implique pour tel ou tel éditeur la transcription de ses partitions, recueils ou méthodes d'apprentissage en Braille ? Serait-il incongru d'inviter l'éditeur en question, à partir de ces chiffres, à contribuer à la transcription ? Pour le savoir, il faudrait évidemment des données très précises sur le marché de l'édition musicale en noir. La question est seulement celle d'une participation des acteurs concernés par le marché à des frais que les " consommateurs " seuls ne peuvent pas supporter.

\section{Quelles réflexions pour la traduction?}

Le musicien malvoyant sera forcément, à un moment ou à un autre, et sans devoir aller pour cela jusqu'aux tropiques, sous le feu des projecteurs, sous les " sunlights "... S'il est professeur, il rencontrera les élèves, leurs parents, leur public. S'il se produit sur scène, il échangera avec un public. Les transcripteurs, eux, restent dans l'ombre, personne ne les voit, alors que leur travail peut être tout bonnement indispensable au musicien. Ils reflètent bien en cela la 
situation de nombreux traducteurs, dont l'invisibilité a maintes fois été soulevée comme un problème, jusqu'à constituer le titre d'un ouvrage de Lawrence Venuti : The Translator's Invisibility.

Michael Cronin estime pourtant que it is, of course, precisely the fact that translation would be perceived to be a "weak tie" that accounts for its importance ; " c'est, bien sûr, précisément parce que la traduction est perçue comme un " lien faible " qu'elle est importante " (Cronin, 2006, p. 141, notre traduction). II explique juste avant que si quelqu'un est à la recherche d'un emploi, par exemple, il est fort probable qu'il l'obtienne grâce à des relations qui ne font pas partie de son entourage proche. En effet, soit sa famille et ses amis vivent dans le même environnement, soit les échanges sont si fréquents que le chercheur d'emploi dispose déjà des informations en leur possession qui lui seraient utiles pour trouver le poste voulu et il ne cherchera pas longtemps. C'est donc l'activation de liens moins forts qui apportera éventuellement l'information pertinente à ce moment-là. Cronin affirme que la traduction est le weak tie qui lie les cultures entre elles.

Le problème réside dans le fait que if the 'weakness' of translation may thus in a sense be accounted as its greatest strength the difficulty is that remuneration, working conditions and status are predicated on perception, not effect ; " si la " faiblesse " de la traduction peut en un sens être considérée comme sa plus grande force, le problème est que la rémunération, les conditions de travail et le statut dépendent de la perception qu'on en a et non de l'effet qu'elle produit " (Cronin, 2006, p. 142). Sans doute les difficultés que nous avons mentionnées dans la partie précédente concernant la rémunération de notre transcriptrice, Marie-Andrée Courjault, peuvent-elles être attribuées en partie à cette invisibilité. Sans doute aussi devrait-on ajouter que le produit qu'elle vend, outre qu'il ne concerne qu'un nombre très restreint de personnes comme nous l'avons dit, est distribué sur un marché qu'on pourrait qualifier de "culturel ", souvent peu rémunérateur y compris pour ses principaux acteurs que sont les musiciens.

Le petit nombre de clients et le prix de revient de la production des transcriptions induisent nécessairement un nombre proportionnel de transcripteurs. Marie-Andrée compte ainsi deux transcripteurs employés à plein temps par l'Association Valentin Haüy à Paris, un transcripteur à l'Institut national des jeunes aveugles pour subvenir en partie aux besoins de l'école, un autre à l'Institut des Hauts Thébaudières de Vertou (44), à proximité de Nantes, et quelques autres personnes qui le font de façon plus ponctuelle. Encore le transcripteur de l'Institut des Hauts Thébaudières ne travaille-t-il pas, au sein de son service de transcription qui emploie six personnes au total, à temps complet sur du contenu musical. Étant donné ce faible nombre, il leur est donc plus facile de s'organiser pour proposer leurs produits au meilleur coût.

Les stratégies sont de deux ordres. Tout d'abord, les transcripteurs s'efforcent de faire jouer le réseau. Ils partagent ainsi leurs catalogues, Marie-Andrée y fait figurer ses projets de transcription pour les six mois suivants, ce qui permet à ses collègues de lui signaler éventuellement qu'ils ont déjà transcrit telle ou telle partition. L'objectif est clair : ne pas transcrire deux fois 
la même partition. Avant de se lancer dans un gros projet, les transcripteurs s'appellent donc pour vérifier qu'il n'est pas déjà sur le métier de quelqu'un d'autre.

D'une certaine manière, c'est ce que font aussi les traducteurs qui constituent des mémoires de traduction et partagent des " glossaires" via des portails tels que proz.com ou gotranslators.com. Le marché n'ayant pas la même taille, le réseau ne peut être aussi efficace et se répartit sur plusieurs plateformes. Mais cela montre, s'il en était encore besoin, et comme on le constate aussi dans les regroupements de traducteurs-interprètes au sein des syndicats, que les collègues sont bien souvent des ressources et des prescripteurs potentiels avant d'être des concurrents. Autrement dit, la solidarité dans un corps de métier peut primer sur la compétition et bénéficier à tous. En outre, on peut considérer que le réseau aura une importance d'autant plus capitale que les marchés de traduction seront restreints, par exemple pour des langues de moindre diffusion.

La deuxième stratégie, comme c'est déjà le cas sur ce type de marché aussi (ar Rouz, 2003, 22), repose sur l'initiative des transcripteurs, qui décident de leur propre chef de transcrire certaines partitions. Selon Marie-Andrée Courjault, en effet, les commandes seules ne couvriraient même pas les frais de papier du CTMBI. En fonction de l'intérêt perçu pour certaines partitions ou méthodes, en fonction des choix pédagogiques des différents conservatoires, elle prend donc l'initiative de transcrire des partitions qui seront ensuite vendues à dix exemplaires maximum chacune mais suffiront, comme nous l'avons dit, à couvrir l'ensemble des frais de fonctionnement de l'association, hors rémunération de la transcriptrice. Marie-Andrée souligne cependant qu'il est extrêmement difficile de suivre l'édition de musique en noir tant elle est foisonnante.

Les traducteurs savent bien que leur production est susceptible d'être une des variables d'ajustement des budgets des grandes entreprises. Elle coûte cher et son importance marketing n'apparaît pas toujours clairement aux comptables. Ils savent aussi que l'Union européenne considère que l'obligation de traduire en français fait obstacle à la libre circulation des marchandises et demande à la France de la supprimer. La première étape de ce processus a déjà eu lieu puisque les entreprises n'ont plus à traduire leurs brevets en français, avec les conséquences que nous connaissons pour nombre de nos collègues.

Quelle voie nous montrent donc les transcripteurs de musique en Braille? Celle de l'initiative? Très bien, mais hormis dans le champ de la littérature, on ne va pas se mettre à traduire des textes techniques, juridiques, marketing ou autres en espérant que des entreprises les achètent. Ce n'est d'ailleurs pas comme cela que procèdent les transcripteurs : ils ne choisissent pas les partitions à transcrire au hasard, ils étudient leur marché. Les traducteurs, eux, étudient-ils suffisamment leurs marchés? Se spécialisent-ils par la force des choses ou dans des secteurs qu'ils ont préalablement sondés?

En admettant qu'ils effectuent ces études de marché, il leur appartient certainement ensuite de faire de la pédagogie sur lesdits marchés, c'est-à-dire d'expliquer aux donneurs d'ordres 
(potentiels) ce qu'apportent leurs productions, en matière de vente peut-être, mais surtout pour la qualité des relations entre fournisseurs et clients, entre fabricants et consommateurs, entre employeurs et employés... Et pour leur fournir des arguments, ne rêverait-on pas d'" études d'impact ", par exemple ? La traductologie a peut-être là à s'approprier les outils d'enquête dont disposent les sociolinguistes, à s'intéresser aussi davantage, suivant en cela la voie esquissée par Anthony Pym (2007), aux données économiques qui fondent les marchés de la traduction, mais encore plus à conquérir les contributions et l'adhésion des traducteurs eux-mêmes.

\section{Conclusion}

La transcription de la musique en Braille, la transcription de textes en Braille, comme la traduction, manquent certainement de visibilité. Combien d'auditeurs des musiciens malvoyants imaginent que leur billet d'entrée au concert peut servir au concertiste à payer des partitions, dont la vente sert à son tour à rémunérer un transcripteur? Plus largement, combien de consommateurs de disques, fussent-ils produits par des majors, se rendent compte que leur achat rémunère des personnes qui travaillent près de chez eux. Certes, les profits de ces grosses entreprises sont vertigineux, les gains de certains artistes sont exagérés et ces déséquilibres conduisent à la défiance, puis au piratage, mais il n'en reste pas moins qu'ils permettent à de nombreux acteurs de l'ombre de travailler.

La musique rassemble indéniablement. Elle crée du lien social, autour d'artistes dans les concerts, autour d'une table à l'écoute d'un disque, sur les pistes de danse, etc. Et même l'auditeur enfermé sous son casque dans les transports en commun finira par partager l'émotion que lui a procuré la musique, du moins s'il n'en devient pas sourd... Pour autant, prendre conscience que c'est ce partage qui est essentiel à nos vies, plutôt que le remplissage de nos étagères de disques, n'a rien d'évident. $A$ fortiori, les rouages de l'économie, son rôle de lien risquent de rester opaques tant que l'essentiel ne sera pas redevenu la première préoccupation des consommateurs que nous sommes.

Les musiciens, en donnant de l'émotion, accèdent au cœur des gens. II nous importe donc que des transcripteurs s'efforcent de "Brailler » la musique, pour qu'ils soient aussi nombreux que possible à brailler, mais en y mettant les formes (!), le désir de plus en plus répandu d'une autre économie, respectueuse de l'humain, et non des profits de quelques-uns. Et pour cela, ils n'ont qu'à exercer leur métier.

II est beaucoup plus improbable que les clients des traducteurs techniques touchent de la même manière leur public. Les traducteurs eux-mêmes gardent cependant la possibilité de prendre la parole, de faire parler d'eux, de leur métier et de son importance dans l'économie et dans les relations interculturelles en général. Certains ne se sentiront pas capables de le 
faire ? Qu'à cela ne tienne ! Exercer leur métier dans de bonnes conditions, échanger avec leurs clients pour établir avec eux des relations de confiance, mutuellement bénéfiques, suffirait déjà à changer l'économie.

En définitive, si la musique adoucit les mœurs et pour qu'elle continue de le faire, elle ne doit certainement pas adoucir le papier des musiciens malvoyants, sous peine de leur ôter en plus la parole ! Et nous avons tous besoin de cette parole musicale...

info@trohadistro.com

Après avoir obtenu son diplôme de traducteur quadrilingue à l'IPLV (Institut de langues vivantes de I'Université Catholique de l'Ouest à Angers), David ar Rouz s'installe en libéral comme traducteur et poursuit en même temps ses études pour terminer sa maîtrise. II choisit alors comme sujet de recherche l'auto-traduction, travaille sous la direction du Professeur Michel Ballard à Arras et interviewe notamment, dans le cadre de ce travail, Nancy Huston et Georges-Arthur Goldschmidt. Passionné dès lors par la recherche en traductologie, il décide de poursuivre par un DEA (master), qui lui permet de traiter cette fois de la traduction en breton et en gallo, les deux langues régionales de Bretagne, sous la direction du Professeur Jean-Yves Urien. Aujourd'hui, quand son activité de traducteur le lui permet, il participe encore occasionnellement à des colloques et rédige des articles, dont plusieurs paraitront en 2009-2010 (la liste en est fournie sur le site http://www.trohadistro.com). David ar Rouz est traducteur (www.trohadistro.com) et musicien (www.myspace.com/flaouiter ou www.transe-en-danse.com).

\section{Pour en savoir plus}

Association Valentin Haüy (AVH), Paris, http://www.avh.asso.fr.

Centre de transcription musicale Braille par informatique (CTMBI), Saint-Nazaire, http://ctmbi.free.fr.

Institut des Hauts Thébaudières, Vertou (44), http://www.thebaudieres.org.

Musica Saint-Nazaire, "La musique en Braille ", http://musica-stnazaire.com/dossiers/braille.htm. 


\section{Bibliographie sommaire}

AR ROUZ, David. Entretiens avec Marie-Andrée Courjault. 24 février et 27 mars 2009.

AR ROUZ, David. La traduction en breton et en gallo : enjeux et difficultés. Mémoire de DEA effectué à l'Université de Haute-Bretagne (Rennes 2), sous la direction du Professeur Jean-Yves Urien. 2003.

BENASAYAG, Miguel et SZTULWARK, Diego. Du contre-pouvoir. Traduit de l'espagnol par Anne Weinfeld. Paris : La Découverte, 2000.

CRONIN, Michael. Translation and Identity. Oxon: Routledge, 2006.

GAGNEPAIN, Jean. Leçons d'introduction à la Théorie de la Médiation. Anthropo-logiques 5. Louvain-la-Neuve : Peeters, 1994.

GAGNEPAIN, Jean. Du vouloir dire. Traité d'épistémologie des sciences humaines. I. Du Signe, De l'Outil. Paris : Livre \& Communication, 1990.

LADMIRAL, Jean-René. Traduire : théorèmes pour la traduction. Saint-Amand : Gallimard, 1994 (première édition : la Petite Bibliothèque Payot, $n^{\circ} 366,1979$ ).

PEETERS, Jean. La médiation de l'étranger. Une sociolinguistique de la traduction. Arras: Artois Presses Université, 1999.

PYM, Anthony. "Globalization and the Politics of Translation Studies", in Meta, vol. 51, n 4, Presses de l'Université de Montréal : Montréal, décembre 2006, pp. 744-757.

VEILHAN, Jean-Claude. Les règles de l'interprétation musicale à l'époque baroque. Paris: éditions Alphonse Leduc, 1977. 